(Supporting Information)

\title{
Atomic Force Microscopy Study of Helical Poly(phenylacetylene)s on Mica Substrate
}

Shin-ichiro Sakurai, Kenji Kuroyanagi, Kazuhide Morino, Masashi Kunitake, and Eiji Yashima*

Department of Molecular Design and Engineering, Graduate School of Engineering,

Nagoya University, Chikusa-ku, Nagoya 464-8603, Japan and Department of Applied Chemistry

\& Biochemistry, Faculty of Engineering, Kumamoto University, Kumamoto 860-8555, Japan

Polymerization. Polymerization was carried out according to Scheme S-1. Poly((4carboxyphenyl)acetylene)s (poly-1L and poly-1H) were prepared by the polymerization of (4-

Scheme S-1. Synthesis of poly-1L, poly-1H, and poly- $(R)-2$

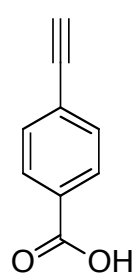

1

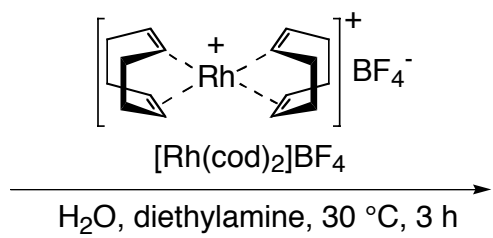

$\mathrm{H}_{2} \mathrm{O}$, diethylamine, $30^{\circ} \mathrm{C}, 3 \mathrm{~h}$

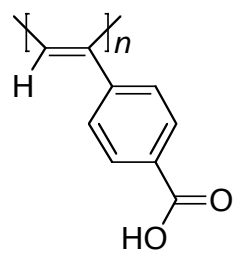

poly-1 L

$$
M_{\mathrm{n}}=3.3 \times 10^{4}, M_{\mathrm{w}} / M_{\mathrm{n}}=2.8
$$<smiles>C#Cc1ccc(C(=O)O)cc1</smiles>

1

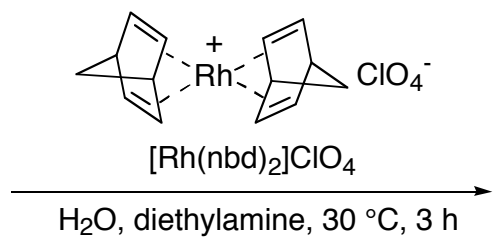

$\mathrm{H}_{2} \mathrm{O}$, diethylamine, $30^{\circ} \mathrm{C}, 3 \mathrm{~h}$

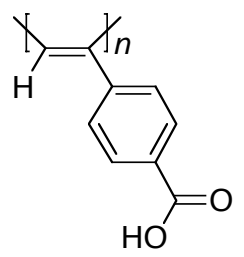

poly-1H

$$
M_{\mathrm{n}}=1.3 \times 10^{5}, M_{\mathrm{w}} / M_{\mathrm{n}}=4.3
$$

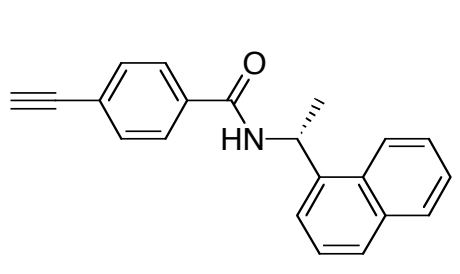

(R)-2
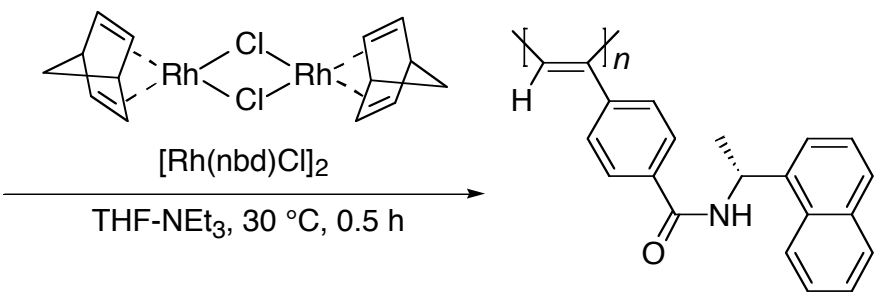

poly- $(R)-2$

$$
M_{\mathrm{n}}=3.0 \times 10^{5}, M_{\mathrm{w}} / M_{\mathrm{n}}=1.8
$$


carboxyphenyl)acetylene (1) according to the previously reported method using a water-soluble rhodium catalyst in water. ${ }^{1}$ The molecular weights of the polymers were estimated as its methyl ester by size exclusion chromatography (SEC) with polystyrene standards using chloroform or tetrahydrofuran (THF) as the eluents. Poly- $(R)-2$ was synthesized according to the previously reported method by polymerization of the corresponding monomer $((R)-2)$ with $[\mathrm{Rh}(\mathrm{nbd}) \mathrm{Cl}]_{2}$ (nbd: norbornadiene) as a catalyst in THF. ${ }^{2}$ The molecular weight of poly-(R)-2 was determined by SEC with poly(ethylene oxide) and poly(ethylene glycol) standards in DMF containing 10 $\mathrm{mM}$ lithium chloride as the eluent.

AFM Measurements. AFM images were obtained in tapping mode using a Nanoscope IIIa (Digital Instruments, Inc. Santa Barbara, CA). The AFM measurements were performed at ambient conditions (in air at ca. $25^{\circ} \mathrm{C}$ ) using silicon cantilevers with a spring constant of ca. 40 $\mathrm{N} / \mathrm{m}$, a tip radius of $5-10 \mathrm{~nm}$, and a resonance frequency of about $300 \mathrm{kHz}$. The instrument was equipped with the Extender Electronics Module (Digital Instruments) to provide simultaneously height and phase images. All the images were collected with the maximum available number of pixels (512) in each direction. The Nanoscope image processing software was used for image analysis.

Sample Preparation. Stock solutions of poly-1L and poly-1H in DMSO (0.5 and 0.05 $\mathrm{mg} / \mathrm{mL})$, poly-( $R$ )-2 in DMF (0.05 mg/mL and $0.025 \mathrm{mg} / \mathrm{mL})$, and poly-1L and poly-1H (0.5 and $0.05 \mathrm{mg} / \mathrm{mL})$ in $0.34 \mathrm{M}$ DMSO solution of $(R)$-Nap were prepared. The stock solutions were deposited on freshly cleaved mica or a highly oriented pyrolytic graphite (HOPG) substrates, the 
solutions were blown off simultaneously with a stream of nitrogen, and the substrates were dried in vacuo overnight before measurements.

CD Measurements. Stock solutions of poly-1H $(6 \mathrm{mg} / \mathrm{mL})$ and $(R)-\mathrm{Nap}(135 \mu \mathrm{L} / 2 \mathrm{~mL})$ in DMSO and poly- $(R)-2(5 \mathrm{mg} / \mathrm{mL})$ in DMF were prepared in a 2-mL flask equipped with a stopcock. A $0.4-\mathrm{mL}$ aliquot of the stock solution of poly-1H was transferred to a flask, and to this was added $0.4-\mathrm{mL}$ of the stock solution of $(R)$-Nap. A $100 \mu \mathrm{L}$ aliquot of the stock solution of poly- $(R)-2$ was transferred to a flask, and the solution was diluted with DMF, giving a 0.5 $\mathrm{mg} / \mathrm{mL}$ solution of poly-(R)-2. The absorption and CD spectra were recorded at ambient temperature $\left(24-26^{\circ} \mathrm{C}\right)$. Figure $\mathrm{S}-2$ shows the CD spectra of poly-1H- $(R)$-Nap complex and $\operatorname{poly}-(R)-2$.

Molecular Modeling and Calculations. Molecular modeling and molecular mechanics calculation were conducted with the Dreiding force field (version 2.21) as implemented in Cerius $^{2}$ software (version 3.8; Molecular Simulation Inc., Burlington, MA, USA) running on an Indigo $^{2}$-Extreme graphics workstation (Silicon Graphics). ${ }^{3}$ The polymer model (20 mer) of poly$(R)-2$ was constructed using a Polymer Builder module in Cerius ${ }^{2}$. Charges on atoms of poly- $(R)-$ 2 were calculated using charge equilibration (Qeq) in Cerius ${ }^{2}$; total charge of the molecule was zero. The starting main chain conformation of a polymer model was defined as the double bond geometry (cis or trans) and a conformation of a rotational single bond. The double bond geometry was fixed to cis and the initial dihedral angle of a single bond from planarity could be varied. Since poly- $(R)-2$ showed an intense CD in the UV-visible of the polymer backbone 
region, chiral side groups of poly-( $R)-\mathbf{2}$ could control a helical state to one-handedness in excess. ${ }^{2}$ However, a preferable helical conformation of poly-(R)-2 (right- or left-handed helix) has not yet been determined, so that we assume that poly- $(R)-2$ may have a right-handed helix and the initial dihedral angle was set to $150^{\circ}$ (transoid) on the basis of the calculated structure of poly((4-(tbutyldimethylsiloxy)phenylacetylene) whose pendant group was similar in bulkiness to that of poly-(R)-2. ${ }^{4}$ The constructed model (20 mer) was optimized by the conjugate gradient method. The energy minimization was continued until the root-mean-square (rms) value became less than $0.1 \mathrm{kcal} \mathrm{mol}^{-1} \AA^{-1}$. The average dihedral angles of the double and single bonds from planarity were $163.7 \pm 5.5^{\circ}$ and $-5.6 \pm 2.1^{\circ}$, respectively. The obtained right-handed helical structure of poly-(R)-2 is shown in Figure 1C.

\section{References}

(1) Saito, M. A.; Maeda, K.; Onouchi, H.; Yashima, E.; Macromolecules 2000, 33, 4616-4618.

(2) Morino, K.; Maeda, K.; Yashima, E. Macromolecules 2003, 36, 1480-1486.

(3) (a) Mayo, S. L.; Olafson, B. D.; Goddard, W. A., III J. Phys. Chem. 1990, 94, 8897-8909.

(b) Rappé, A. K.; Goddard, W. A., III J. Phys. Chem. 1991, 95, 3358-3363. (c)

Castonguay, L. A.; Rappé, A. K.; Casewit, C. J. J. Am. Chem. Soc. 1991, 113, 7177-7183.

(4) Yashima, E.; Huang, S.; Matsushima, T.; Okamoto, Y. Macromolecules 1995, 28, 4184-4193. 


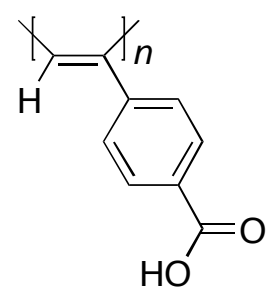

poly-1H<smiles>CC(N)c1cccc2ccccc12</smiles>

(R)-Nap

$$
M_{\mathrm{n}}=1.3 \times 10^{5}, M_{\mathrm{w}} / M_{\mathrm{n}}=4.3
$$

$$
\text { [poly-1H] }=0.05 \mathrm{mg} / \mathrm{mL} \quad \text { - HOPG - }
$$

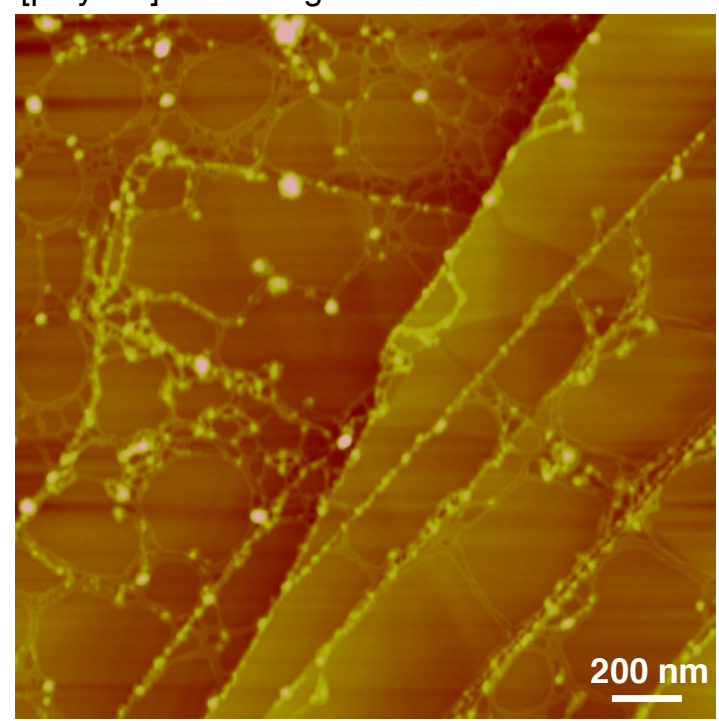

$$
\text { [poly-1H] }=0.05 \mathrm{mg} / \mathrm{mL}
$$

$$
[(R)-\mathrm{Nap}]=0.34 \mathrm{M} \quad-\mathrm{HOPG} \text { - }
$$
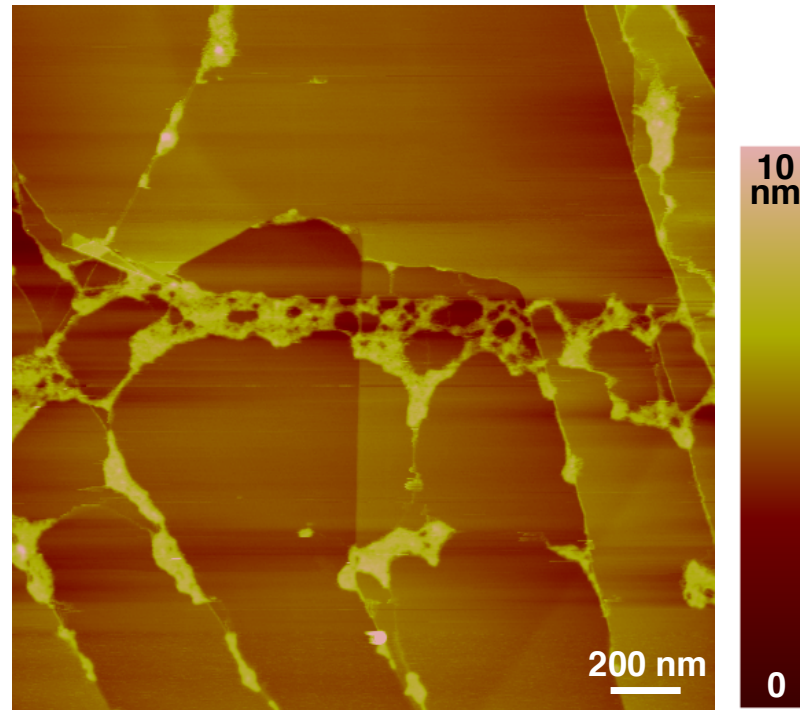

Figure S-1. AFM images of poly-1H with and without $(R)-$ Nap on HOPG. 


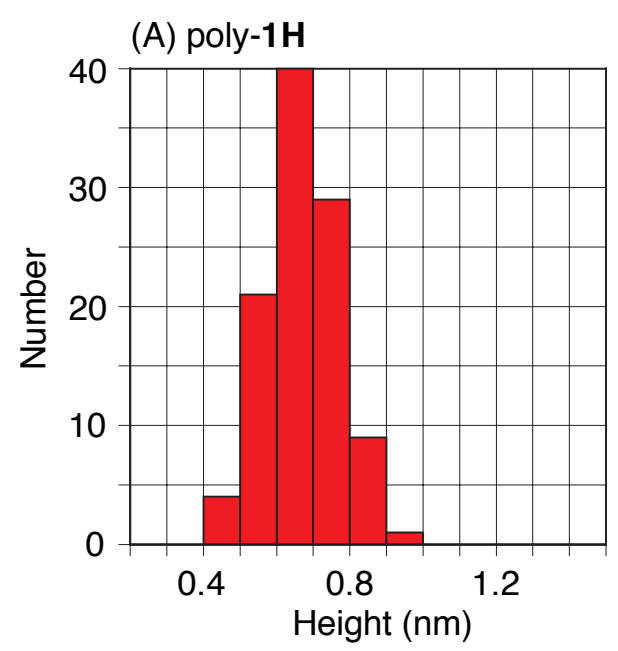

$0.67 \pm 0.10 \mathrm{~nm}$

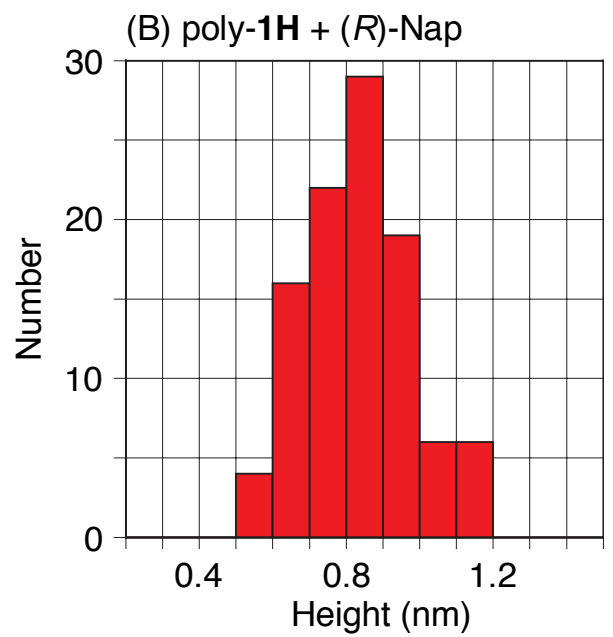

$0.83 \pm 0.15 \mathrm{~nm}$

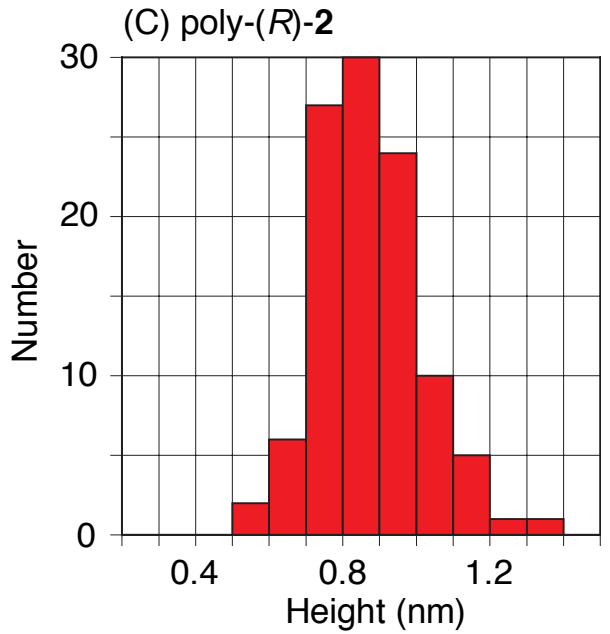

$0.88 \pm 0.14 \mathrm{~nm}$

Figure S-2. Histograms of height distribution of poly-1H (A), poly-1H- $(R)$-Nap complex $(\mathrm{B})$, and poly- $(R)-2(\mathrm{C})$. The average heights of polymers were evaluated for an assembly of ca. 100 cross-section profiles. 

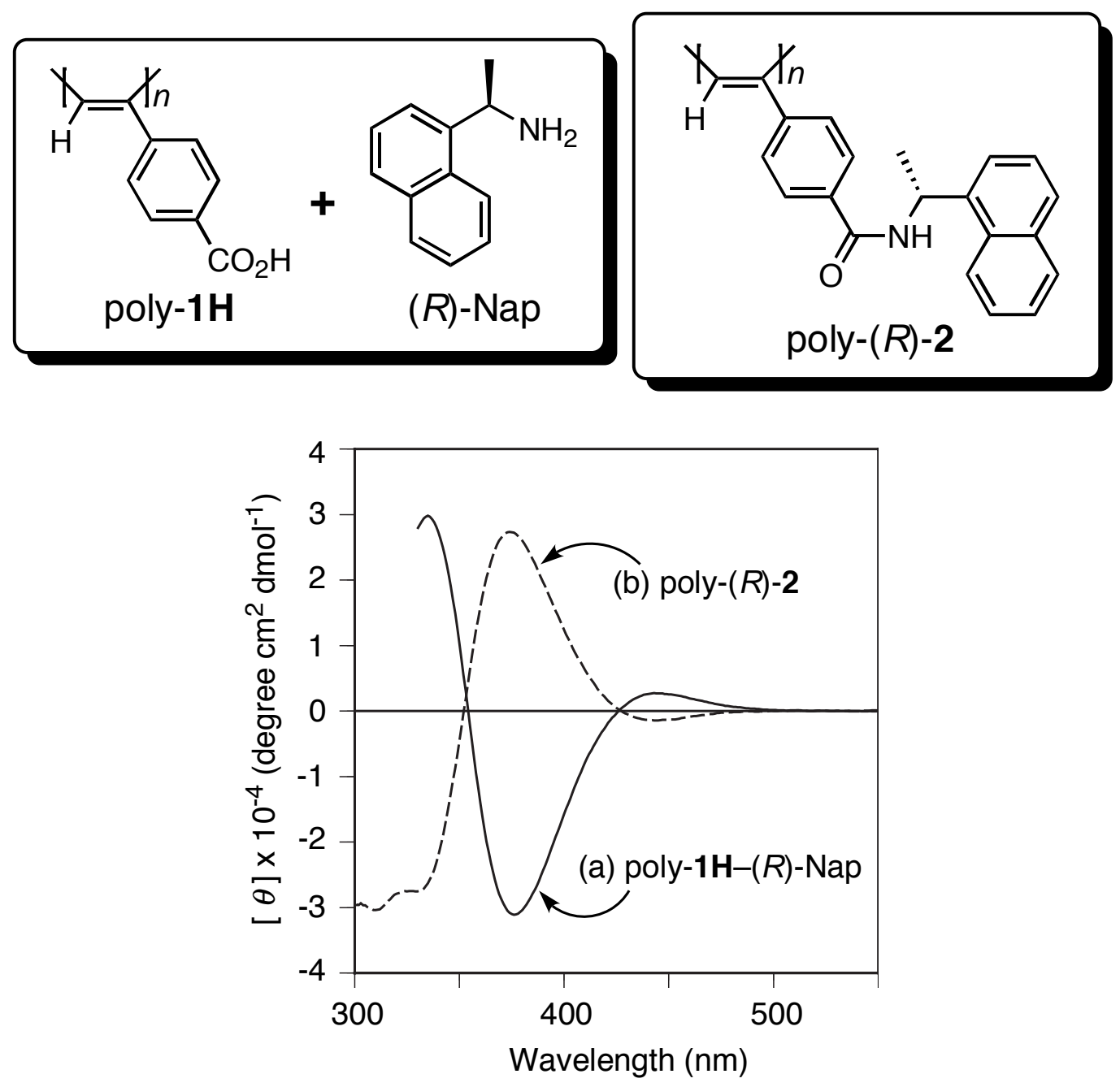

Figure S-3. CD spectra of poly-1H and poly- $(R)-2$ in $0.34 \mathrm{M}$ DMSO solution of ( $R$ )-Nap (a) and DMF (b), respectively. 


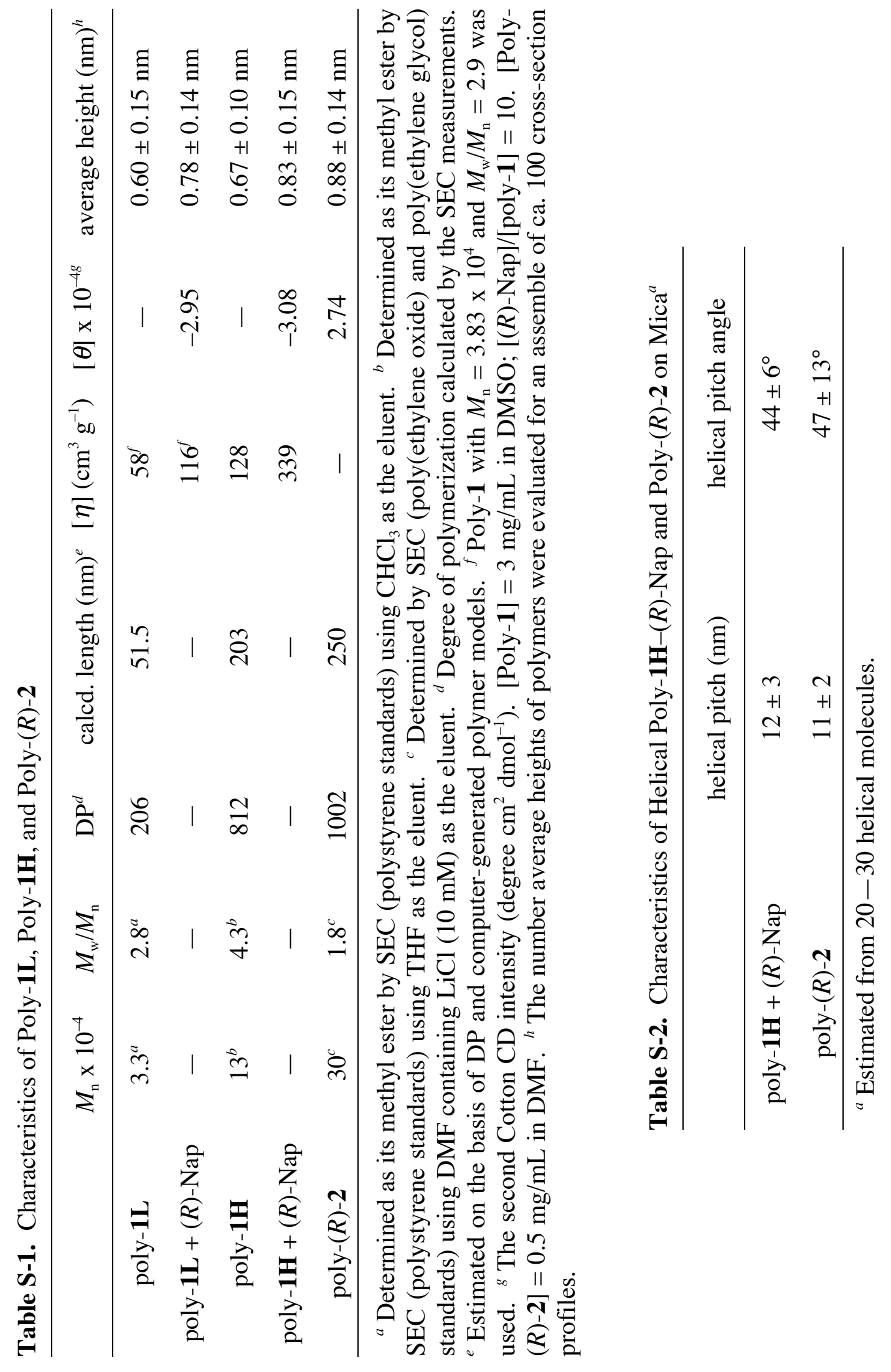




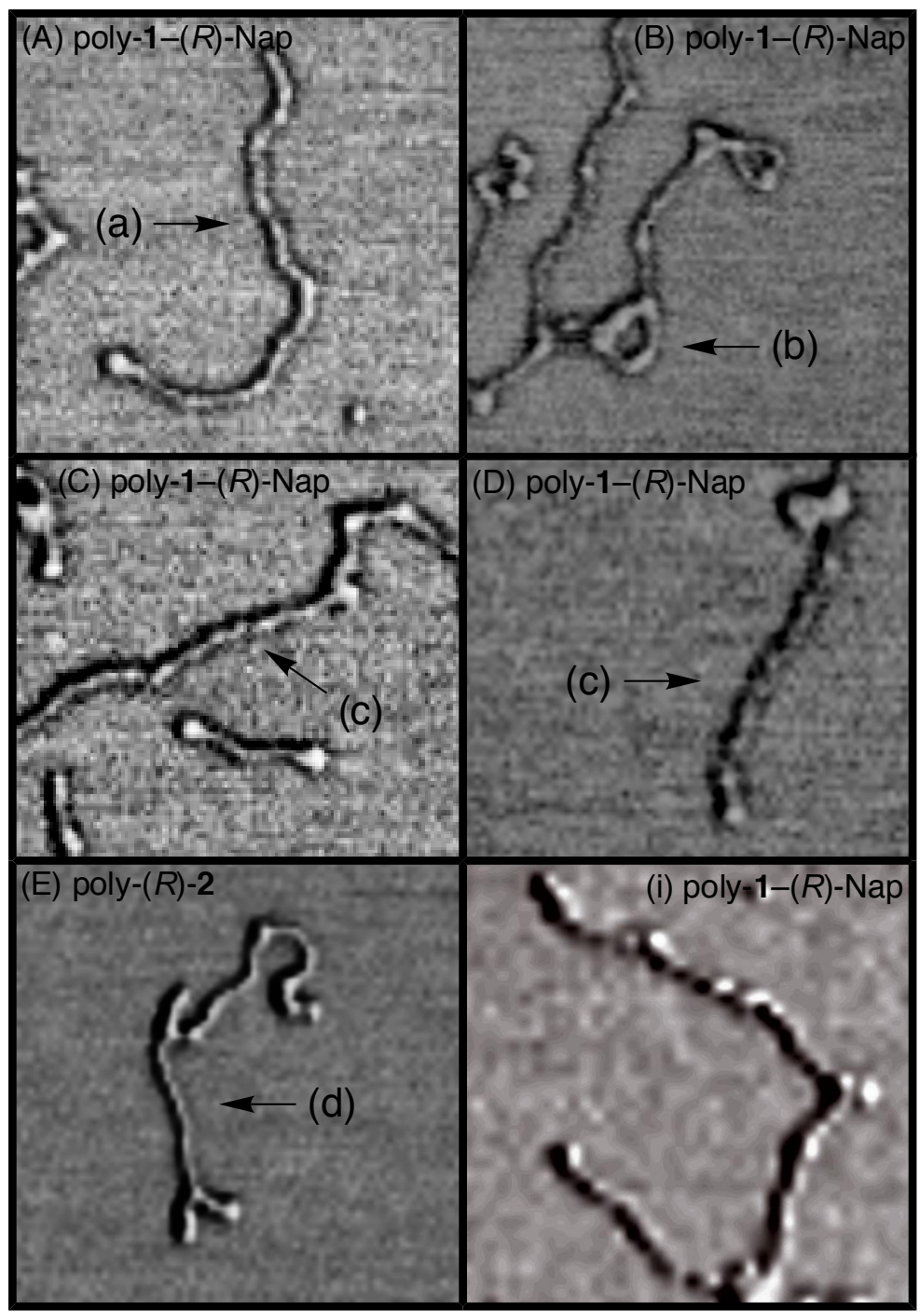

Figure S-4. Expanded AFM phase images of poly-1H- $(R)$-Nap complex $(\mathrm{A}-\mathrm{D})$ and poly- $(R)-\mathbf{2}$ on mica $(\mathrm{E})$. These images are from Figures $4 \mathrm{~B}$ and $4 \mathrm{D}$, respectively. Image (i) is taken from a different area. 